\title{
Papers
}

\section{Survey of unlicensed and off label drug use in paediatric wards in European countries}

\author{
Sharon Conroy, Imti Choonara, Piero Impicciatore, Angelika Mohn, Henrik Arnell, Anders Rane, \\ Carmen Knoeppel, Hannsjoerg Seyberth, Chiara Pandolfini, Maria Pia Raffaelli, Francesca Rocchi, \\ Maurizio Bonati, Geert 't Jong, Matthijs de Hoog, John van den Anker on behalf of the European \\ Network for Drug Investigation in Children
}

\begin{abstract}
Objective To determine the extent of use of unlicensed and off label drugs in children in hospital in five European countries.

Design Prospective study of drugs administered to children in general paediatric medical wards over four weeks.

Setting Children's wards in five hospitals (one each in the United Kingdom, Sweden, Germany, Italy, and the Netherlands).

Subjects Children aged 4 days to 16 years admitted to general paediatric medical wards.

Main outcome measure Proportion of drugs that were used in an unlicensed or off label manner.

Results 2262 drug prescriptions were administered to 624 children in the five hospitals. Almost half of all drug prescriptions $(1036 ; 46 \%)$ were either unlicensed or off label. Of these 1036, 872 were off label and 164 were unlicensed. Over half of the patients $(421 ; 67 \%)$ received an unlicensed or off label drug prescription. Conclusions Use of off label or unlicensed drugs to treat children is widespread. This problem is likely to affect children throughout Europe and requires European action.
\end{abstract}

\section{Introduction}

Many drugs used to treat children in hospital are either not licensed for use in children or are prescribed outside the terms of their product license (off label prescribing). ${ }^{12}$ Examples of use of off label drugs include diazepam rectal solution in children under 1 year (not licensed for age group), amiloride tablets in any children (formulation), or rectal injection of lorazepam for a child with an acute seizure (route). An example of unlicensed use is the preparation of a suspension from a tablet by the hospital pharmacy.

Considerable concern exists within Europe $^{3}$ and the United States ${ }^{45}$ about the use of unlicensed and off label drugs in children. There is, however, little information available on the extent to which these types of treatments are used. The extent of use of unlicensed and off label drugs in the United Kingdom has been reported in a paediatric intensive care unit, ${ }^{6}$ paediatric medical and surgical wards, ${ }^{2}$ and a neonatal intensive care unit. ${ }^{7}$ We wished to determine the extent of unlicensed and off label drug use in several countries within the European Union. This is important in view of the new European guidance on the clinical investigation of medicinal products in children. ${ }^{8}$

\section{Methods}

We studied a paediatric medical ward in each of the participating centres (Derby, United Kingdom; Uppsala, Sweden; Marburg, Germany; Bergamo, Italy; Rotterdam, Netherlands) prospectively for four consecutive weeks during 1998. The wards in Derby and Bergamo admitted mainly general paediatric patients, with Derby including children who had had surgery. The wards in Marburg and Uppsala had a mixture of general paediatric and respiratory cases (including cystic fibrosis). The ward in Rotterdam had the fewest general paediatric cases, containing children with cardiac, oncological, renal, and respiratory disease. Data on all patients admitted to the ward were collected by the investigator in each centre. The child's age, date of birth, weight, and diagnosis were recorded as well as details of all drugs administered (route of administration, dose, and indication for use). We did not include standard intravenous replacement solutions, flushes of $0.9 \%$ sodium chloride or heparin, blood products, oxygen, or drugs in clinical trials.

We assessed all drugs administered to determine if their use was unlicensed and off label using a previously described classification system. ${ }^{12}$ Categories of unlicensed use were modification of licensed drugs (such as crushing tablets to prepare a suspension); drugs that are licensed but the formulation is manufactured under a special licence (such as a liquid preparation of a drug that is licensed only in tablet form); new drugs available under a special manufacturing licence (such as caffeine injections for apnoea of prematurity); use of chemicals as drugs when no pharmaceutical grade preparation is available; drugs used before a licence has been granted; and imported drugs (drugs imported from a country where they are licensed). Off label use included use of a drug in situations not covered by the product licence or the

\author{
Academic Division \\ of Child Health \\ (University of \\ Nottingham), \\ Derbyshire \\ Children's Hospital, \\ Derby DE22 3NE \\ Sharon Conroy \\ lecturer in paediatric \\ clinical pharmacology \\ Imti Choonara \\ professor \\ Piero Impicciatore \\ research fellow \\ Angelika Mohn \\ research fellow \\ University Hospital, \\ Uppsala, Sweden \\ Henrik Arnell \\ research fellow \\ Anders Rane \\ professor of clinical \\ pharmacology \\ University \\ Children's Hospital, \\ Marburg, Germany \\ Carmen Knoeppel \\ medical student \\ Hannsjoerg \\ Seyberth \\ professor of \\ paediatrics \\ Mario Negri \\ Institute, Milan, Italy \\ Chiara Pandolfini \\ research fellow \\ Maria Pia Raffaelli \\ hospital pharmacist \\ Francesca Rocchi \\ research fellow \\ Maurizio Bonati \\ head of unit \\ continued over
}

BMJ 2000;320:79-82 
Department of Paediatrics

(Erasmus University

Rotterdam), Sophia

Children's Hospital,

Rotterdam,

Netherlands

Geert 't Jong

research fellow

Matthijs de Hoog

consultant in

paediatric critical care

John van den Anker

professor in

paediatrics and

neonatology

Correspondence to:

I Choonara

Imti.choonara@

nottingham.ac.uk

Table 1 Number of patients and prescriptions in each centre

\begin{tabular}{lcccccc} 
& Derby & Uppsala & Marburg & Bergamo & Rotterdam & Total \\
\hline No of patients & 192 & 87 & 85 & 118 & 142 & 624 \\
\hline Age range & 21 days- & 4 days- & 28 days- & 30 days- & 4 days-16 years & 4 days-16 years \\
& 16 years & 15 years & 16 years & 12 years &
\end{tabular}

\begin{tabular}{lcccccc}
\hline Prescriptions & 798 & 185 & 224 & 398 & 657 & 2262 \\
\hline Mean No of prescriptions/patient & 4.2 & 2.1 & 2.6 & 3.4 & 4.6 & 3.6
\end{tabular}

\begin{tabular}{|c|c|c|c|c|c|c|}
\hline ean No of prescriptions/patient & 4.2 & 2.1 & 2.6 & 3.4 & 4.6 & 3.6 \\
\hline $0(\%)$ of prescriptions unlicensed or off label & $239(30)$ & $57(31)$ & $91(41)$ & $264(66)$ & $385(59)$ & $1036(46)$ \\
\hline
\end{tabular}

No $(\%)$ of prescriptions unlicensed 58

$8(4)$

\begin{tabular}{llllll}
\hline No $(\%)$ of prescriptions off label & $181(23)$ & $49(26)$ & $83(37)$ & $263(66)$ & $296(45)$ \\
\hline
\end{tabular}

treatment

Table 2 Five most frequently prescribed drugs (\% of all prescriptions) in each centre

\begin{tabular}{|c|c|c|c|c|c|c|c|c|c|c|}
\hline \multirow[b]{2}{*}{ Rank } & \multicolumn{2}{|c|}{ Derby } & \multicolumn{2}{|c|}{ Uppsala } & \multicolumn{2}{|c|}{ Marburg } & \multicolumn{2}{|c|}{ Bergamo } & \multicolumn{2}{|c|}{ Rotterdam } \\
\hline & Drug & $\%$ & Drug & $\%$ & Drug & $\%$ & Drug & $\%$ & Drug & $\%$ \\
\hline $1 \mathrm{st}$ & Paracetamol & 21 & Paracetamol & 22 & Paracetamol & 9 & Beclometasone & 12 & Paracetamol & 4 \\
\hline 2nd & Ibuprofen & 10 & Cefuroxime & 8 & Cholecalciferol & 8 & Dipyrone & 8 & Heparin & 4 \\
\hline $3 \mathrm{rd}$ & Salbutamol & 5 & Salbutamol & 7 & Cefuroxime & 7 & Salbutamol & 8 & Amphotericin & 3 \\
\hline 4th & Cyclizine & 5 & Ibuprofen & 6 & Salbutamol & 7 & Paracetamol & 7 & Pancreatin & 2 \\
\hline 5 th & Morphine & 4 & Cotrimoxazole & 5 & Xylometazoline & 5 & Amoxycillin & 6 & Spironolactone & 2 \\
\hline
\end{tabular}

Table 3 Five most frequently prescribed off label drugs in each centre

\begin{tabular}{|c|c|c|c|c|c|c|c|c|c|c|}
\hline \multirow[b]{2}{*}{ Rank } & \multicolumn{2}{|c|}{ Derby } & \multicolumn{2}{|c|}{ Uppsala } & \multicolumn{2}{|c|}{ Marburg } & \multicolumn{2}{|c|}{ Bergamo } & \multicolumn{2}{|c|}{ Rotterdam } \\
\hline & Drug & $\mathrm{No}^{*}$ & Drug & No* & Drug & No* & Drug & No* & Drug & $\mathrm{No}^{*}$ \\
\hline $1 \mathrm{st}$ & Cyclizine & $38 / 42$ & Salbutamol & $13 / 13$ & Budesonide & $10 / 12$ & Beclometasone & $47 / 47$ & Heparin & $28 / 28$ \\
\hline 2nd & Salbutamol & $27 / 42$ & Paracetamol & $13 / 41$ & Salbutamol & $8 / 17$ & Salbutamol & $28 / 32$ & Pancreatin & $17 / 17$ \\
\hline $3 r d$ & Morphine & $26 / 33$ & Cotrimoxazole & $4 / 10$ & Xylometazoline & $8 / 15$ & Paracetamol & $26 / 28$ & Spironolactone & $17 / 17$ \\
\hline 4th & Ipratropium & $15 / 15$ & Betamethasone & $3 / 5$ & Paracetamol & $7 / 20$ & Betamethasone & $21 / 38$ & Frusemide & $16 / 18$ \\
\hline 5 th & Diazepam & $13 / 13$ & Acetylcysteine & $2 / 4$ & Chloral hydrate & $5 / 5$ & Amoxycillin & $18 / 23$ & Tobramycin & $15 / 16$ \\
\hline
\end{tabular}

${ }^{*}$ Number of off label presciptions/total number of prescriptions for drug in centre.

summary of product characteristics-that is, at a different dose or frequency, in different clinical indications, in different age groups, administration by an alternative route, or in a formulation not approved for use in children.

The primary reference sources for determining licensed indications were the Association of the British Pharmaceutical Industry's Data Sheet Compendium in the United Kingdom; the Swedish Physician's Desk Reference 1998 in Sweden; the Rote Liste 1996 and FachInfo compact disc (1997) in Germany; the Informatore Farmaceutico 1998 (national formulary) and technical leaflets in Italy; and the Repertorium 98/99 and Farmacotherapeutisch Kompas 1998 in the Netherlands.

\section{Results}

A total of 624 children were admitted to the general paediatric wards in the five participating centres and received 2262 drug prescriptions (table 1). The prescribing habits in the five centres differed greatly. Paracetamol was the most widely prescribed drug and analgesic in four of the five centres. Dipyrone was frequently used in Italy only. Salbutamol and cefuroxime were both widely used (table 2). Almost half of all drug prescriptions (1036) were either unlicensed or off label (table 1). Many more prescriptions were off label (872) than unlicensed (164). The results were remarkably similar in Derby, Uppsala, and Marburg. Use of unlicensed and off label drugs was greatest in Bergamo and Rotterdam, with Bergamo having the highest percentage of off label prescriptions $(66 \%)$ and Rotterdam the highest percentage of unlicensed prescriptions (14\%).

Over half of the children $(421 ; 67 \%)$ received an unlicensed or off label drug prescription during their stay in hospital. Analgesics and bronchodilators were among the five most frequently prescribed off label drugs in four centres (table 3). The commonest category of off label drug use was dose and frequency in three centres (Uppsala, Marburg, and Bergamo), accounting for more than half of off label use. In the other two centres (Derby and Rotterdam) dose and frequency accounted for $31-32 \%$ of off label drug use. The main category for off label drug use in Rotterdam was formulation. Formulation was also an important category in Bergamo but not in the other centres. Age was the commonest category of off label drug use in Derby (table 4). Table 5 shows examples of off label drug use. In Bergamo 53\% of the children who received beclometasone were under 12 months old, although it is licensed only for children aged 2 years and over in Italy.

\section{Discussion}

The drug use in the five paediatric wards differed. This is not surprising as each of the wards had different subspecialty interest and prescribing habits are different within each country. ${ }^{9}$ Unlicensed drug use was highest in Rotterdam, which had the highest number of patients with complex diseases. Many of these children received drugs that are not available in a paediatric formulation and therefore had to be modified by the 
pharmacy department to make them suitable for administration to children. Stability data are rarely available for such products, which are rendered unlicensed by this modification. Dipyrone is no longer available in many European countries because of the risk of agranulocytosis. ${ }^{10}$ It is, however, widely used in Italy. About half of the children in each of the five countries received drugs that are either unlicensed or off label.

It is concerning that most bronchodilator drug prescriptions for children in hospital with asthma are off label, since this is a common condition for which there has been considerable research. The efficacy of bronchodilators in children under the age of 2 years is variable, especially in infants under the age of 12 months. A particular problem was the widespread use of inhaled corticosteroids in children under the age of 2 years (off label for age and dose); few data exist on the effect of inhaled corticosteroids on growth suppression in this age group. Studies are required to determine whether the off label use of bronchodilators is justified by good scientific evidence.

The most common reasons for off label use were that the medicine was prescribed at a different dose or frequency, in a different formulation, or in an age group for which it had not been licensed. There were also some children who received the drug for a different indication or by an alternative route. It is ironic that it is children who are most likely to receive medicines that are either unlicensed or used off label since the regulations for the licensing of medicines were introduced after cases of drug toxicity in the developing fetus (thalidomide) and newborn infant (chloramphenicol induced grey baby syndrome). ${ }^{3}$

Not all off label drug use is inappropriate. Drug toxicity is more likely with aminoglycosides if they are used in neonates as recommended by the manufacturers at intervals of 8-12 hours rather than at longer intervals. In many cases, however, the risk of off label drug use is not known because there are inadequate data. A recent study has shown that adverse drug reactions are an important problem in children after unlicensed or off label drug prescriptions. ${ }^{11}$

\section{Key messages}

- Many drugs are not tested in children, which means that they are not specifically licensed for use in children

- Licensed drugs are often prescribed outside the terms of the product license (off label) in relation to age, indication, dose of frequency, route of administration, or formulation

- Over two thirds (67\%) of 624 children admitted to wards in five European hospitals received drugs prescribed in an unlicensed or off label manner

- $39 \%$ of the 2262 drug prescriptions given to children were off label

- The problem of off label and unlicensed drug prescribing in children is a European problem that requires European action
Table 4 Number (percentage) of off label prescriptions in each category for five centres

\begin{tabular}{lccccc} 
Category & Derby & Uppsala & Marburg & Bergamo & Rotterdam \\
\hline Dose and frequency & $66(32)$ & $53(88)$ & $59(61)$ & $255(58)$ & $96(31)$ \\
\hline Age & $79(39)$ & $1(2)$ & $23(24)$ & $33(7)$ & $18(6)$ \\
\hline Indication & $36(17)$ & $4(7)$ & $7(7)$ & $25(6)$ & $13(4)$ \\
\hline Route & $24(12)$ & $2(3)$ & $3(3)$ & $49(11)$ & $4(1)$ \\
\hline Formulation & 0 & 0 & $5(5)$ & $80(18)$ & $176(58)$ \\
\hline Total & 205 & 60 & 97 & 442 & 307 \\
\hline
\end{tabular}

Table 5 Examples of off label drug use

\begin{tabular}{ll} 
Drug & Off label use \\
\hline Beclometasone & Used in infants under 12 months. Licensed for 2 years and over in Italy \\
\hline Fluticasone & $250 \mu$ twice daily in 4 year old. Maximum dose $100 \mu \mathrm{g}$ twice daily \\
\hline Trimeprazine & $\begin{array}{l}\text { Used as sedative in child with pneumonia. Licensed for urticaria, pruritus, and } \\
\text { pre-anaesthetic medication }\end{array}$ \\
\hline Rifampicin & Used for enzyme induction in infant with biliary atresia \\
\hline Salbutamol & Used two hourly (12 times daily). Licensed for 4 times daily \\
\hline Tobramycin & Used once daily in neonate. Licensed for twice daily \\
\hline
\end{tabular}

\section{Reducing the risk}

The new European guidance on the clinical investigation of medicinal products in children encourages pharmaceutical companies that wish to introduce new products to investigate these in children when clinically appropriate. Changes have also been made in the United States to encourage pharmaceutical companies to carry out clinical trials in children. These changes in regulations may improve knowledge for new products, although a recent study found little improvement in new drugs licensed in Europe. ${ }^{12}$ However, a major problem remains with many existing drugs commonly used in children. Health professionals concerned about the lack of information regarding the use of drugs in children are in a difficult situation. They need to raise awareness of the problem in society as a whole without causing undue anxiety among parents.

To ensure that children are not exposed to unnecessary risks, controlled clinical trials are required to determine the most appropriate dose in children of different ages. A mechanism and infrastructure needs to be established to determine who will fund these trials. The European Network for Drug Investigation in Children has been established to try to improve this situation. ${ }^{13}$ We feel that the European Union, national departments of health, and politicians as well as the European Medicines Evaluation Agency must take a more proactive role in getting drugs tested in children. If they fail to do so, children will continue to be denied the same rights as adults in relation to receiving treatment with drugs that have been fully tested.

Contributors: IC initiated and designed the study, supervised the collection of data, analysed the data, and was involved in writing the paper. SC designed the data collection forms, coordinated and was involved in collecting and analysing the data, and was involved in writing the paper. AM, MPR, FR, GJ, and MH were involved in analysis of the data and in writing the paper. PI, HA, $\mathrm{AR}, \mathrm{CK}, \mathrm{HS}, \mathrm{CP}, \mathrm{MB}$, and JA were involved in the design of the study, helped collect the data, analysed the data, and were involved in writing the paper.

Funding: None.

Competing interests: IC, AR, HS, and JA attended a round table meeting of experts on the use of new medicines in children organised by EMEA in 1997. IC has a grant from the Medicines Control Agency in the United Kingdom in relation to drug reaction surveillance in children. 
1 Turner S, Nunn AJ, Choonara I. Unlicensed drug use in children in the UK. Paediatr Perinat Drug Ther 1997;1:52-5.

2 Turner S, Longworth A, Nunn AJ, Choonara I. Unlicensed and off label drug use in paediatric wards: prospective study. BMJ 1998:316:343-5.

3 Choonara I, Dunne J. Licensing of medicines. Arch Dis Child 1998;78:402-3.

4 Nahata MC. Licensing of medicines for children in the USA. Paediatr Perinat Drug Ther 1997;1:50-1.

5 Cote CJ, Kauffman RE, Troendle GJ, Lambert GH. Is the "therapeutic orphan" about to be adopted? Pediatrics 1996;98:118-23.

6 Turner S, Gill A, Nunn T, Hewitt B, Choonara I. Use of "off-label" and unlicensed drugs in paediatric intensive care unit. Lancet 1996;347:549-50.

7 Conroy S, McIntyre J, Choonara I. Unlicensed and off label drug use in the neonate. Arch Dis Child Fetal Neomatal Ed 1999/80:F142-5.

8 EMEA. Note for guidance on clinical investigation of medicinal products in children London: EMEA, 1997.
9 Bonati M. Epidemiological evaluation of drug use in children. J Clin Pharmacol 1994;34:300-5.

10 International Agranulocytosis and Aplastic Anemia Study. Risk of agranulocytosis and aplastic anemia. A first report of their relation to drug use with special reference to analgesics. JAMA 1986;256:1749-57.

11 Turner S, Nunn AJ, Fielding K, Choonara I. Adverse drug reactions to unlicensed and off-label drugs on paediatric wards: a prospective study. Acta Paediatrica 1999;88:965-8.

12 Impicciatore P, Choonara I. Status of new medicines approved by the European Medicines Evaluation Agency regarding paediatric use. $\mathrm{Br} \mathrm{J}$ Clin Pharmacol 1999;48:15-8.

13 Bonati M, Choonara I, Hoppu K, Pons G, Seyberth H. Closing the gap in drug therapy. Lancet 1999;353:1625.

(Accepted 1 November 1999)

\section{Difficulties with anonymous shortlisting of medical school applications and its effects on candidates with non-European names: prospective cohort study}

Andrew B Lumb, Andy Vail

\begin{abstract}
School of Medicine, University of Leeds, Worsley Medical and Dental Building, Leeds LS2 9NL

Andrew B Lumb sub dean for admissions

Andy Vail senior medical statistician

Correspondence to A B Lumb:medal@ leeds.ac.uk
\end{abstract}

BMJ 2000;320:82-5

\begin{abstract}
Objective To assess the feasibility of anonymous shortlisting of applications for medical school and its effect on those with non-European names.

Design Prospective cohort study.

Setting Leeds school of medicine, United Kingdom. Subjects 2047 applications for 1998 entry from the United Kingdom and the European Union.

Intervention Deletion of all references to name and nationality from the application form.
\end{abstract}

Main outcome measures Scoring by two admissions tutors at shortlisting.

Results Deleting names was cumbersome as some were repeated up to 15 times. Anonymising application forms was ineffective as one admissions tutor was able to identify nearly $50 \%$ of candidates classed as being from an ethnic minority group. Although scores were lower for applicants with non-European names, anonymity did not improve scores. Applicants with non-European names who were identified as such by tutors were significantly less likely to drop marks in one particular non-academic area (the career insight component) than their European counterparts.

Conclusions There was no evidence of benefit to candidates with non-European names of attempting to blind assessment. Anonymising application forms cannot be recommended.

\section{Introduction}

In the United Kingdom there is huge competition to study medicine, with in excess of 13000 applications through the Universities and Colleges Admissions Service for just over 4000 places. A series of recent studies has found that the likelihood of success is less among applicants from ethnic minority groups than among white applicants. ${ }^{1-6}$ A study based on 1991 entry indicated that the situation was improving. ${ }^{5}$ Even so, when seven other mainly academic aspects of the application were taken into account not being from an ethnic minority group remained a significant predictor of success. A recent study looking at all home applicants for entry in 1996 and 1997 found a greater disadvantage for applicants from ethnic minority groups than previously. ${ }^{6}$ This study was, however, limited for technical reasons by not being able to include data on GCSE grades, which form a large part of the selection process and which were important predictors of success in previous studies. ${ }^{5}$

Most unsuccessful applicants are rejected solely on assessment of their application form-that is, at the 'shortlisting' stage before being invited for interview. It is during shortlisting that students from ethnic minority groups are believed to be disadvantaged. ${ }^{5}$ The application form contains no explicit reference to the applicant's ethnic background, so it seems likely that any discrimination must be based on the applicant's name. For this reason it has been suggested that the whole of the shortlisting process be performed anonymously. ${ }^{5}$

We decided to assess the feasibility of assessing forms anonymously within the current admissions system of the Universities and Colleges Admissions Service. In addition, we assessed the impact of doing so on the shortlisting system we have used at Leeds school of medicine for the past four years.

\section{Methods}

Shortlisting process

Our shortlisting process involves each application form being assessed separately by two of three admissions tutors (including AL). A score from zero to 20 points is awarded made up of four components including career insight (4 points), non-academic activities (6 points), academic profile (4 points), and suitability for a medical career as described by the confidential reference (6 points). When assessing applications, admissions tutors are unaware of the other selector's score. The sum of the two scores then forms the sole basis of the decision to reject, accept, or interview the 